\title{
Case report: whole genome sequencing based investigation of maternal-neonatal listeriosis in Sichuan, China
}

Lijuan Luo ${ }^{1,3}$, Xi Chen², Michael Payne ${ }^{3}$, Xiaolong Cao ${ }^{4}$, Yan Wang ${ }^{1}$, Jie Zhang ${ }^{2}$, Jianping Deng ${ }^{2}$, Hong Wang ${ }^{2}$, Zhengdong Zhang ${ }^{2}$, Qun Li ${ }^{2}$, Ruiting Lan ${ }^{3^{*}}$ and Changyun Ye

\begin{abstract}
Background: Neonatal listeriosis is a rare but severe disease manifesting as septicemia and central nervous system (CNS) infections with a high fatality rate of around 20 to 30\%. Whole genome sequencing (WGS) is a promising technique for pathogen identification and infection source tracing with its high resolution.

Case presentation: A case of neonatal sepsis with listeriosis was reported with positive blood culture for Listeria monocytogenes. The case was investigated to confirm the vertical transmission of the infection and identify the potential food source of the maternal L. monocytogenes infection using WGS. L. monocytogenes was isolated from the neonate's blood sample the day after caesarean delivery and from the mother's genital and pudenda swab samples 5 days and 13 days after caesarean delivery. WGS showed that the isolate from the neonate was identical to the genome type of the isolates from the mother, with only one of the 4 isolates from the mother differing by one single nucleotide polymorphism (SNP). By WGS, one L. monocytogenes isolate from a ready-to-eat (RTE) meat sample in the patients' community market shared the same sequence type but was ruled out as the cause of infection, with 57 SNP differences to the strain causing the maternal-neonatal infection. The food isolate also carried a novel plasmid pLM1686 that harbored heavy metal resistance genes. After caesarean section, the mother was treated with a third generation cephalosporin which L. monocytogenes is naturally resistant to, which may explain why genital and pudenda swabs were still culture-positive for L. monocytogenes 13 days after delivery.

Conclusions: Genital swab culture for L. monocytogenes had been informative in the diagnosis of maternal listeriosis in this case. The high resolution of WGS confirmed the maternal-neonatal transmission of L. monocytogenes infection and ruled out the L. monocytogenes contaminated RTE meat from the local market as the direct source of the mother's infection.
\end{abstract}

Keywords: Case report, Maternal-neonatal listeriosis, Whole genome sequencing, Plasmid

\footnotetext{
* Correspondence: yechangyun@icdc.cn; r.lan@unsw.edu.au

${ }^{1}$ State Key Laboratory of Infectious Disease Prevention and Control, National

Institute for Communicable Disease Control and Prevention, Collaborative

Innovation Center for Diagnosis and Treatment of Infectious Diseases,

Chinese Center for Disease Control and Prevention, Beijing 102206, China

${ }^{3}$ School of Biotechnology and Biomolecular Sciences, University of New

South Wales, Sydney, NSW 2052, Australia

Full list of author information is available at the end of the article
}

(c) The Author(s). 2019 Open Access This article is distributed under the terms of the Creative Commons Attribution 4.0 International License (http://creativecommons.org/licenses/by/4.0/), which permits unrestricted use, distribution, and reproduction in any medium, provided you give appropriate credit to the original author(s) and the source, provide a link to the Creative Commons license, and indicate if changes were made. The Creative Commons Public Domain Dedication waiver (http://creativecommons.org/publicdomain/zero/1.0/) applies to the data made available in this article, unless otherwise stated. 


\section{Background}

Neonatal listeriosis is an infection caused by Listeria monocytogenes, leading to sepsis and meningitis with a high fatality rate around 20 to $30 \%$ [1, 2]. Early-onset neonatal listeriosis (defined as infection in neonates with less than 7 days of age) is mostly caused by vertical transmission from the mother $[3,4]$. L. monocytogenes can traverse the placenta and infect the fetus, resulting in abortion, stillbirth, premature birth and neonatal listeriosis [5]. However, maternal listeriosis is relatively mild in severity manifesting as flu-like symptoms (such as fever, myalgia, nausea and vomiting) with $30 \%$ of maternal listeriosis patients being asymptomatic [1]. Thus, it is difficult to recognize maternal-neonatal listeriosis due to the mild or asymptomatic maternal listeriosis before it develops into life threatening conditions of neonatal bacteremia or meningitis through vertical transmission. While neonatal sepsis and meningitis cases caused by $L$. monocytogenes are relatively rare (2 to $7 \%$ of infections) [6,7], it is vital to increase clinicians' awareness on maternal-neonatal listeriosis for early diagnosis and effective targeted treatment, especially in countries such as China where listeriosis is less recognized and there are no surveillance systems in place [8].

L. monocytogenes is a common foodborne pathogen [9]. Outbreaks may be traced back to the food source and whole genome sequencing (WGS) has been demonstrated as a powerful tool for source tracing of L. monocytogenes outbreaks [10]. In this study, we investigated a case of maternal-neonatal listeriosis using WGS to confirm vertical transmission and to identify the potential food source of the maternal $L$. monocytogenes infection.

\section{Case presentation}

A 21-year-old pregnant woman without a significant medical history, was admitted to the local community hospital with lower abdominal pain at 36 weeks gestation in Sichuan, China. Her axillary temperature was $37.7^{\circ} \mathrm{C}$. Physical examination found that her labium majus pudendi was red and swollen, with moderate pain. White blood cell (WBC) count was $13.1 \times 10^{9} / \mathrm{L}$. A female afebrile baby was born prematurely by cesarean section because of intrauterine distress. After the cesarean, the mother was treated intravenously with cefoperazone/sulbactam sodium $3.0 \mathrm{~g}$ in $100 \mathrm{~mL}$ saline twice a day and was discharged from hospital after 10 days (Fig. 1a). The neonate showed signs of asphyxia and meconium aspiration syndrome after birth and was transferred to a higher tier city hospital. Considering that the mother presented with signs of infection prior to delivery, the neonate's WBC count was $26.7 \times 10^{9} / \mathrm{L}$ and C-reactive protein was $60.8 \mathrm{mg} / \mathrm{L}$, neonatal sepsis was strongly suspected. The neonate was empirically treated with sodium penicillin of 260,000 units (around 100,000 units/kg/ dose) and ceftazidime $134 \mathrm{mg}(50 \mathrm{mg} / \mathrm{kg} / \mathrm{dose})$, both of which were administered intravenously every $12 \mathrm{~h}$. Blood culture was performed when the neonate entered the hospital and L. monocytogenes was isolated and identified 4 days later. Lumbar puncture and cerebrospinal fluid culture were not performed, therefore neonatal meningitis cannot be excluded. After 13 days' treatment, the neonate's C-reactive protein level returned normal and blood culture was negative for L. monocytogenes. The neonate was then discharged from the hospital (Fig. 1a).

This listeriosis case was reported to the local Center for Disease Control and Prevention, and the following investigations were carried out. Blood and milk samples, vaginal fornix and pudenda swabs from the mother were collected and cultured for L. monocytogenes in the community hospital (Fig. 1a). L. monocytogenes was isolated from the vaginal fornix and pudenda swabs while blood culture and breast milk culture were negative. Culture for L. monocytogenes from stool samples from the mother was not performed. The patient, who had been discharged home, was followed-up at home 13 days after the delivery. Vaginal fornix and pudenda swabs were again collected from the mother and were positive for $L$. monocytogenes. An extensive source tracing of the $L$. monocytogenes infection was performed. A face-to-face interview was conducted to determine the mother's food exposure history during the previous 1 month prior to the premature delivery. The mother had a history of consuming ice cream, ready-to-eat (RTE) meat and salads. Food and environmental samples from her home (30 samples including the fridge food and fridge surface swabs, kitchen and bathroom environmental swabs), RTE meat, RTE salads, raw pork, beef and frozen chicken samples from the patient's community market (52 samples), and ice cream in the local grocery store (3 samples) were collected (Fig. 1a). One RTE meat sample and one frozen chicken sample from the community market were positive for L. monocytogenes while all other samples were negative.

All clinical isolates ( 2 neonate isolates and 5 mother isolates) and two food isolates of L. monocytogenes were subjected to multi-locus sequence typing (MLST) [11]. All human isolates and one RTE meat isolate belonged to sequence type 87 (ST87), one raw chicken isolate belonged to ST9. The chicken isolate was not closely related to the human cases as it belonged to a different ST while the RTE meat isolate had the same ST and may be related which required further assessment using a higher resolution method.

In order to confirm the vertical transmission of listeriosis and resolve the relationship between the RTE food isolate and the patient isolates, the genomes of all 8 ST87 isolates were sequenced by Illumina sequencing. Using the complete genome ICDC-LM188 (GenBank accession No. 


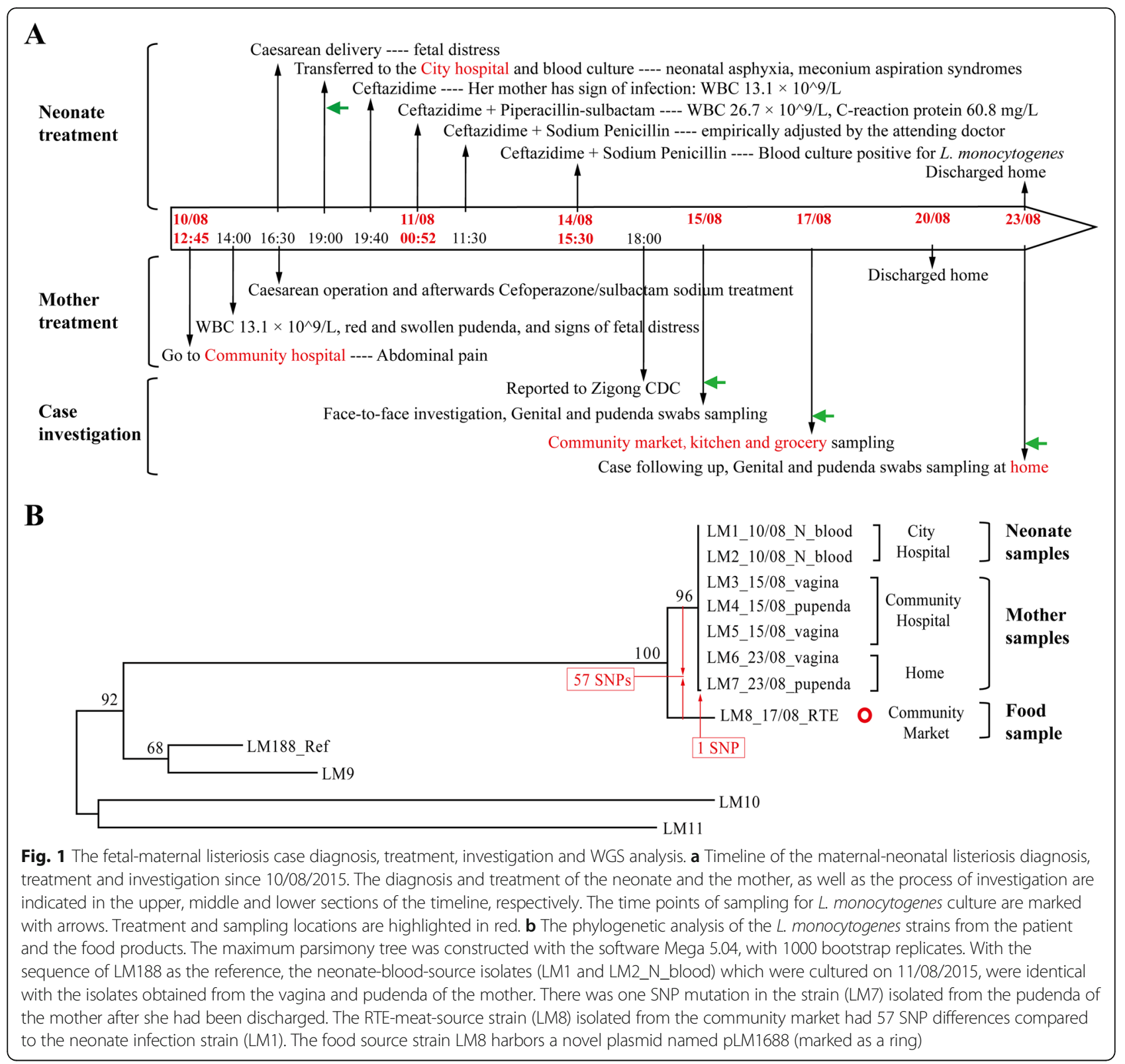

CP015593.1) as the reference, SNPs were called using Burrows-Wheeler Aligner (BWA-MEM) method [12]. At least 10 reads and a coverage of $70 \%$ were required to call a SNP. All human isolates from the neonate and the mother were identical except for one isolate from the mother (LM7). LM7, isolated from the second pudenda swab (13 days after the caesarean), differed from the other clinical isolates by a single SNP (Fig. 1B). However, the RTE meat $L$. monocytogenes isolate showed 57 SNP differences (56 single base mutations and one single base deletion) from the main genome type of the clinical isolates. Among the 57 SNP differences, there were 11 located in the core genome of L. monocytogenes (Additional file 1: Table S1). Considering the mutation rate of 0.4 SNPs per core genome per year [13], the human isolates would have been separated from the food isolate around 27.5 years ago (Fig. 1b). By core-genome MLST (cgMLST) analysis, L. monocytogenes isolates from the baby and the mother (LM1 to LM7) all belonged to the same cgMLST type: L1SL87-ST87-CT5541. Note that LM7 from the mother has one allele difference to the other isolates (LM1 to LM6) reflecting the single SNP difference observed, but both were assigned the cgMLST type as L. monocytogenes strains with no more than 7 alleles are considered to be epidemiologically linked and are assigned to the same cgMLST type [13], although this cutoff is debatable. The RTE meat strain belonged to L1-SL87-ST87-CT5542 (Additional file 1: Table S2). Therefore, the $L$. 
monocytogenes contaminated RTE meat from local market was not the source of the mother's infection.

Analysis of virulence genes showed that the isolates from the patients and RTE food, all of which belonged to ST87, harbored the newly discovered Listeria pathogenicity island 4 (LIPI-4) [14], and intact InlA and inlB genes that encode the invasive proteins internalin $A$ and internalin B respectively [15] were present.

Additionally, the RTE meat isolate (LM8) carried a novel plasmid, named pLM1686. By assembling the raw Illumina reads of LM8 using SKESA v2.3, we identified pLM1686 as a circular plasmid [16]. The plasmid was annotated using Prokka v1.12, and compared with reported plasmids of $L$. monocytogenes using Roary v3.11.2 with an identity of 70\% at nucleotide level as cut-off $[17,18]$. Plasmid pLM1686 was found to be most similar to the previously reported Listeria plasmid pLMR479a [19]. Among the 91 annotated genes of pLM1686, 79 genes were present in pLMR479a, including the heavy metal resistance genes (Fig. 2) (Additional file 1: Table S3). The remaining 12 genes of pLM1686 that were absent in pLMR479a, were present in either plasmid pLM6179 or pLM5578 (Fig. 2) (Additional file 1: Table S3) [20, 21].

\section{Discussion and conclusions}

Maternal-fetal listeriosis is a rare but severe infection [4]. L. monocytogenes is one of the few foodborne pathogens that can be vertically transmitted to the unborn fetus [2]. In this case, WGS confirmed the vertical transmission with the neonatal isolate being identical to maternal isolates. L. monocytogenes is thought to reach the placenta most commonly via hematogenous spread from the gastrointestinal tract to the placenta [5]. However, the exact route of transmission for this case was uncertain. Blood culture from the mother was negative for $L$. monocytogenes and the placenta was not sampled. Therefore, the hematogenous route cannot be confirmed. On the other hand, vaginal fornix swabs and pudenda swabs were positive. It is possible that $L$. monocytogenes may have reached the fetus via an ascending route from the genital tract, which has rarely been reported [22], resulting in fetal infection and distress. The infected fetus displayed signs of distress, which increases the risk for passage of meconium, aspiration and neonatal asphyxia. As such, the infant showed evidence of meconium aspiration syndrome and

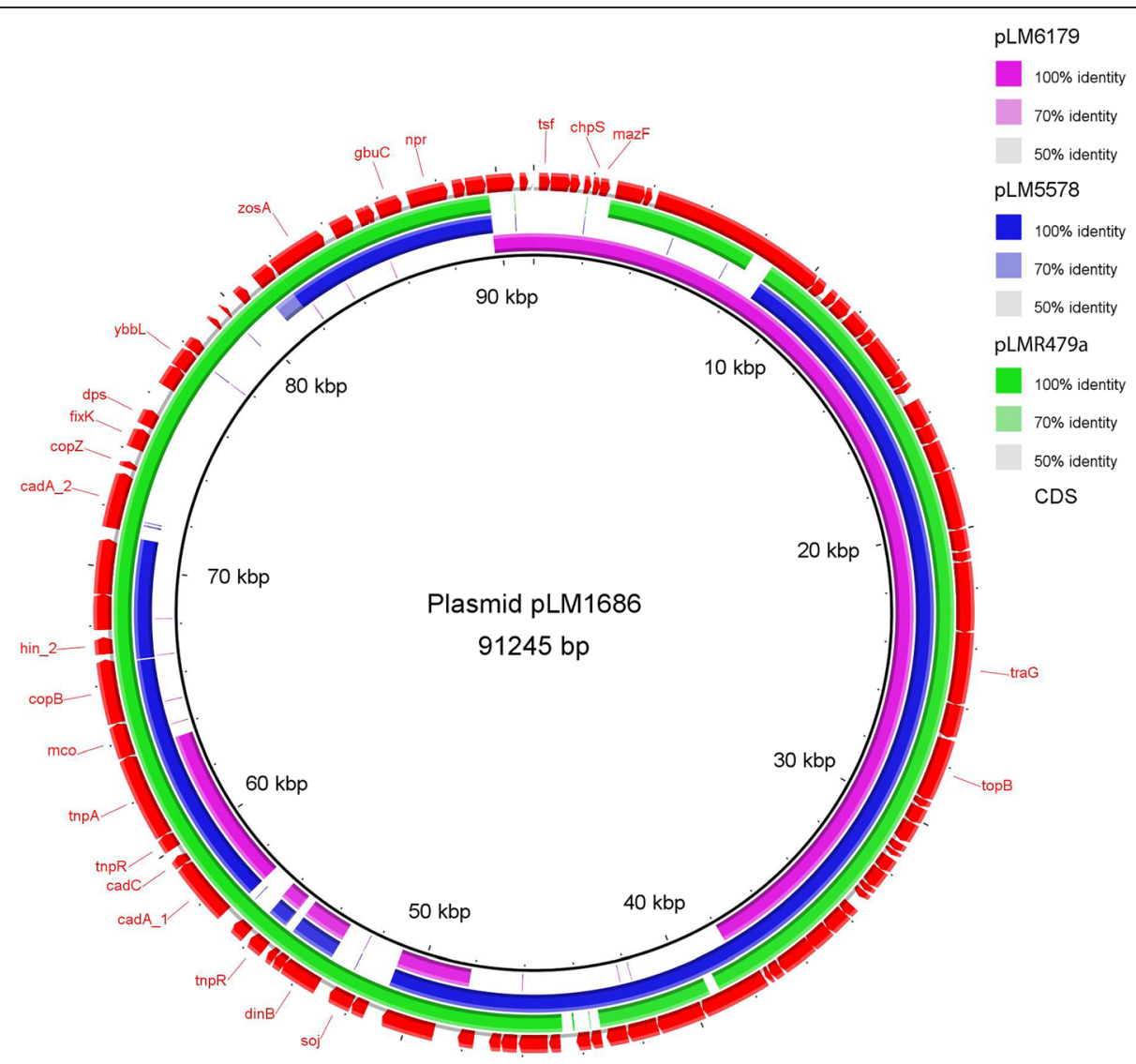

Fig. 2 The plasmid comparison using BRIG with the plasmid pLM1686 as the reference. The newly found pLM1686 (being set as the reference) was compared to previously described plasmids pLM1679, pLMR5578 and pLM479a, represented in purple, blue and green, respectively. The outer red ring refers to the annotation of pLM1686 with the known genes indicated 
neonatal asphyxia after birth, indicating a dangerous course of infection.

Diagnosis of maternal and neonatal listeriosis as early as possible is critical to improve prognosis [23]. Maternal listeriosis cases may only show mild symptoms with $30 \%$ cases being asymptomatic [1]. Therefore, maternal listeriosis cases can easily be overlooked until the fetus has developed listeriosis or the mother presents with obstetric symptoms. In this case, the pregnant woman showed symptoms of colpitis, and vaginal/pudenda swabs were culture-positive for L. monocytogenes. This highlights that for suspected maternal-fetal listeriosis cases, non-invasive cervical/genital swabs culture for L. monocytogenes may assist in diagnosis of fetal listeriosis, in addition to maternal blood culture $[5,15]$. A recent study reported that $55 \%$ of maternal blood samples and $26 \%$ of maternal cervical or vaginal swabs in maternal-neonatal listeriosis cases were culture positive for $L$. monocytogenes [4]. Placenta samples and gastric aspirates from neonates showed the highest rate of positive culture for L. monocytogenes in maternal-fetal listeriosis cases [4]. Therefore, during the delivery, placental tissue for L. monocytogenes culture, would be most valuable in assessing whether neonatal listeriosis resulted from vertical transmission.

The recommended first line empirical therapy for suspected early onset neonatal sepsis in many countries is ampicillin plus gentamicin which cover $L$. monocytogenes $[24,25]$, while in China penicillin combined with a third-generation cephalosporin are commonly used [26]. For the empirical therapy of suspected meningitis, ampicillin plus third-generation cephalosporin are recommended, and cerebrospinal fluid culture is required to guide appropriate therapy [25]. In this case, the neonatal sepsis was empirically treated with penicillin $\mathrm{G}$ and ceftazidime, which was appropriate because neonatal meningitis was suspected. It should be noted the patient's family objected to lumbar puncture for cerebrospinal fluid analysis on the baby and meningitis was not confirmed. Since L. monocytogenes is sensitive to penicillin $\mathrm{G}$ but naturally resistant to cephalosporins [2], ceftazidime should have been removed from the neonate's treatment regimen after $L$. monocytogenes infection was confirmed from blood culture.

Treatment for maternal listeriosis is mostly focused on the antepartum treatment of pregnant women to prevent fetal/neonatal listeriosis [2, 27]. Postpartum treatment for mothers after delivery has rarely been discussed in the literature, as the mother can recover without treatment [2]. In this case, the mother was given cefoperazone/sulbactam after the cesarean which was inappropriate treatment for listeriosis, as L. monocytogenes is naturally resistant to cefoperazone (a cephalosporin) and penicillin is the primary antibiotic for the treatment of listeriosis [2, 28]. Although the patient made an uneventful recovery, it was noteworthy that the vaginal fornix and pudenda swabs were still positive for L. monocytogenes 13 days after delivery. It has been reported that among the mothers that didn't receive appropriate antibiotic therapy for listeriosis (although only 10 cases), more than half of their infants developed late onset disease [4]. This case highlights why more attention should be paid to the appropriate postpartum treatment of maternal listeriosis.

The mother was most likely initially infected by a single strain. Among the isolates from the mother and the neonate, there was only one SNP mutation observed in the pudenda-swab isolate which was isolated 13 days after the cesarean delivery. This mutation may have been present in the earlier period but not sampled or it could also have appeared within the 13-day period. Based on the mutation rate of L. monocytogenes $(2.5 \times$ $10^{-7}$ substitution per site per year) [13], a mutation may arise in as little as 30 days. The single SNP mutation in the core genome may have occurred during the infection period of the mother. But it is also possible that the mother initially infected by two related strains of L. monocytogenes.

In an attempt to trace the source of infection, food and environment samples from the patient's home and local market were sampled. One RTE meat L. monocytogenes isolate obtained from the patient's community market was found to have the same ST as the patients' isolates. However, by WGS analysis, the RTE meat isolate differed from the clinical isolates with 57 SNP differences and belonged to a different cgMLST type from the case, which would have diverged approximately 27.5 years ago. Thus, the RTE food is not a direct infection source of this case. This is not surprising as ST87 is prevalent in China and considerable genomic diversity must have developed [9]. Nevertheless, many reported listeria outbreaks were caused by RTE food, which were consumed without heating [29]. From 2017 to 2018, there was a large scale listeriosis outbreak of 1060 cases in Africa caused by one brand of RTE meat with a mortality rate of $27 \%$ [30]. In Canada, two historical outbreaks were confirmed to be caused by RTE crabmeat through WGS analysis [31]. From 2015 to 2016 in Italy, there was one listeriosis outbreak due to L. monocytogenes contaminated processed pork products [32]. During 2015 to 2017, one cross-border listeriosis outbreak occurred in Denmark and France was caused by coldsmoked salmon from a third European Union country [33]. In our case investigation, the presence of L. monocytogenes in the RTE meat in the patient's community market was a health risk to the local consumers. Our study highlighted that case investigation can help identify L. monocytogenes contaminated food products and prevent further infections. 
This WGS-based analysis showcased the power for tracing the infection source, due to its high discriminatory power. This study applied two major genomic tools (SNP analysis and cgMLST typing) for the WGS analysis of the $L$. monocytogenes isolates $[12,13]$. The results of both methods were concordant with each other. The standardized nomenclature of cgMLST enables comparison between different studies [13].

Interestingly, a novel plasmid pLM1686 was found in the $L$. monocytogenes isolate from the RTE meat sample while the human isolates did not harbor any plasmids. Plasmid pLM1686 was most closely related to plasmid pLMR479a. Both plasmids harbored heavy metal resistance genes (e.g. cadA1, $\operatorname{cadA2}, \operatorname{cad} C$ and $\operatorname{cop} B$ ) and other genes that are related with environmental adaption [34, 35]. Plasmid pLMR479a is common in the globally prevalent ST8 strains of L. monocytogenes [36, 37]. A recent study has confirmed that pLMR479a contributed to stress tolerance of $L$. monocytogenes against disinfectants, acidity, salinity and oxidation [38]. ST87 is a common type of $L$. monocytogenes in either clinic infections or food contaminations in Asia [39-41]. Plasmid pLM1686 may enhance the environmental survival of ST87 L. monocytogenes and further studies are required.

Maternal-neonatal listeriosis is a rare but severe vertical transmissible disease caused by the foodborne pathogen $L$. monocytogenes. Genital swab culture for L. monocytogenes had been informative in the diagnosis of maternal listeriosis in this case although blood culture was negative. Placenta tissue or placental smear culture should be performed for premature delivery with no known cause and can be of assistance in confirming Listeria infection and route of transmission. As L. monocytogenes is generally resistant to third generation cephalosporins, they should not be used for treating maternal-neonatal listeriosis. WGS showed high resolution source tracing and confirmation of maternal neonatal transmission of $L$. monocytogenes infection.

\section{Supplementary information}

Supplementary information accompanies this paper at https://doi.org/10. 1186/s12879-019-4551-9.

Additional file 1: Table S1. Location of the 57 SNPs between the patient's isolate and the RTE food isolate. The SNP differences and the location of the 57 SNPs between the RTE food isolate (LM8) and the patient's isolate (LM1) were listed using LM188 (GenBank accession No. CP015593.1) as the reference. The SNPs on the core genes of $L$. monocytogenes were also indicated. Table $\mathbf{S 2}$. The cgMLST types of the patients and food source L. monocytogenes strains isolated at different time point in this case investigation. The cgMLST types of the patients and food source L monocytogenes were assigned by the cgMLST database (https:/bigsdb.pasteur.fr), with allele profiles offered) Table S3. The annotation of plasmid pLM1686 and the distribution of the genes in the other three online plasmids (identity $70 \%$ ). The circular sequence of the plasmid pLM1686 was assembled with SKESA V2.3 using the raw reads of Illumina sequencing. The plasmid sequence was annotated with Prokka v1.12. The annotation of the pLM1686 were compared with the online plasmids of $L$. monocytogenes using Roary v3.11.2 with the identity of $70 \%$ as the cut-off. Plasmid pLM1686 was found to be most similar to the previously reported Listeria plasmid pLMR479a sharing 79 genes. The remaining 12 unshared genes of pLM1686 with pLMR479a, were present in either plasmid pLM6179 or pLM5578.

\section{Abbreviations}

cgMLST: core genome MLST; CNS: Central nervous system; LIPI-4: Listeria pathogenicity island 4; LM: Listeria monocytogenes; MLST: Multilocus sequence typing; pLM: Plasmid of Listeria monocytogenes; RTE: Ready-to-eat; SNP: Single nucleotide polymorphism; ST: Sequence type; WBC: White blood cell; WGS: Whole genome sequencing

\section{Acknowledgements}

We thank the patient for providing consent to publish this case report. We thank the curators of the Institute Pasteur MLST and genome databases for curating the data and making them publicly available at https://bigsdb. pasteur.fr.

\section{Authors' contributions}

$L L, X C, Q L, H W$ and CYY designed the study; $L L, Y W$ and XLC performed the experiments; XC, JZ, XLC, ZDZ, JPD collected the epidemiological data; $L I L, X C, Y W$ collected the microbiological data; $L L, M P, R L$ analyzed the data; $L J L, M P, R L, C Y Y$ wrote the manuscript. All authors had read and approved the final manuscript.

\section{Funding}

This work was supported by State Key Laboratory of Infectious Disease Prevention and Control, China CDC (No. 2015SKLID507, 2015SKLID306, 2018SKLID801), and National Institute for Communicable Disease Control and Prevention, China CDC (No. 2017ZZKTB09). Lijuan Luo was supported by a UNSW scholarship. The funders played no roles in the design of the study and collection, analysis, and interpretation of data and in writing the manuscript.

\section{Availability of data and materials}

The genome assembly sequences of the strains and the plasmid pLM1686 were deposited at GenBank under the Bioproject of PRJNA503772 and PRJNA447903. All data generated or analysed during this study are included in this published article and its supplementary information files.

Ethics approval and consent to participate Not applicable.

\section{Consent for publication}

Written informed consents were obtained from the patient for publication of this study.

\section{Competing interests}

The authors declare that they have no competing interests.

\section{Author details}

${ }^{1}$ State Key Laboratory of Infectious Disease Prevention and Control, National Institute for Communicable Disease Control and Prevention, Collaborative Innovation Center for Diagnosis and Treatment of Infectious Diseases, Chinese Center for Disease Control and Prevention, Beijing 102206, China. ${ }^{2}$ Zigong Center for Disease Control and Prevention, Zigong 643000, China. ${ }^{3}$ School of Biotechnology and Biomolecular Sciences, University of New South Wales, Sydney, NSW 2052, Australia. ${ }^{4}$ Beijing Changping Institute for Tuberculosis Prevention and Treatment, Beijing 102206, China.

Received: 24 February 2019 Accepted: 3 October 2019 Published online: 26 October 2019

\section{References}

1. Pucci L, Massacesi M, Liuzzi G. Clinical management of women with listeriosis risk during pregnancy: a review of national guidelines. Expert Rev Anti-Infect Ther. 2018;16(1):13-21.

2. Allerberger $F$, Huhulescu S. Pregnancy related listeriosis: treatment and control. Expert Rev Anti-Infect Ther. 2015;13(3):395.

3. Shane AL, Sánchez PJ, Stoll BJ. Neonatal sepsis. Lancet. 2017;390:10104. 
4. Charlier C, Perrodeau É, Leclercq A, Cazenave B, Pilmis B, Henry B, Lopes A Maury MM, Moura A, Goffinet F. Clinical features and prognostic factors of listeriosis: the MONALISA national prospective cohort study. Lancet Infect Dis. 2017:17(5):510.

5. Janakiraman $\mathrm{V}$. Listeriosis in pregnancy: diagnosis, treatment, and prevention. Rev Obstet Gynecol. 2008;1(4):179.

6. Anand V, Holmen J, Neely M, Pannaraj PS, Dien BJ. Closing the Brief Case: Neonatal Meningitis Caused by Listeria monocytogenes Diagnosed by Multiplex Molecular Panel. J Clin Microbiol. 2016;54(12):3075.

7. Lu Q, Zhou M, Tu Y, Yao Y, Yu J, Cheng S. Pathogen and antimicrobial resistance profiles of culture-proven neonatal sepsis in Southwest China, 1990-2014. J Paediatr Child Health. 2016;52(10):939.

8. Li W, Bai L, Fu P, Han H, Liu J, Guo Y. The Epidemiology of Listeria monocytogenes in China. Foodborne Pathog Dis. 2018;15(8):459.

9. Wang H, Luo L, Zhang Z, Deng J, Wang Y, Miao Y, Zhang L, Chen X, Liu X, Sun S. Prevalence and molecular characteristics of Listeria monocytogenes in cooked products and its comparison with isolates from listeriosis cases. Front Med. 2018;12(1):104.

10. Conrad A, Sabol A, Silk BJ, Tarr C, Trees E, Carleton H, Besser J, Roache K, Jackson KA, Gould LH, et al. Implementation of Nationwide Real-time Whole-genome Sequencing to Enhance Listeriosis Outbreak Detection and Investigation. Clin Infect Dis. 2016;63(3):104.

11. Ragon M, Wirth T, Hollandt F, Lavenir R, Lecuit M, Le Monnier A, Brisse S. A new perspective on Listeria monocytogenes evolution. PLoS Pathog. 2008; 4(9):e1000146

12. Li H, Durbin R. Fast and accurate short read alignment with BurrowsWheeler transform. Bioinformatics. 2009;25(14):e1000146.

13. Moura A, Criscuolo A, Pouseele H, Maury MM, Leclercq A, Tarr C, Björkman JT, Dallman T, Reimer A, Enouf V. Whole genome-based population biology and epidemiological surveillance of Listeria monocytogenes. Nat Microbiol. 2017;2(2):16185

14. Maury MM, Tsai Y-H, Charlier C, Touchon M, Chenal-Francisque V, Leclerca A, Criscuolo A, Gaultier C, Roussel S, Brisabois A. Uncovering Listeria monocytogenes hypervirulence by harnessing its biodiversity. Nat Genet. 2016;48(3):308

15. Disson O, Grayo S, Huillet E, Nikitas G, Langa-Vives F, Dussurget O, Ragon M, Le Monnier A, Babinet C, Cossart P, et al. Conjugated action of two speciesspecific invasion proteins for fetoplacental listeriosis. Nature. 2008:455:1114.

16. Souvorov A, Agarwala R, Lipman DJ. SKESA: strategic k-mer extension for scrupulous assemblies. Genome Biol. 2018;19(1):153.

17. Seemann T. Prokka: rapid prokaryotic genome annotation. Bioinformatics. Bioinformatics. 2014;30:14

18. Page AJ, Cummins CA, Hunt M, Wong VK, Reuter S, Holden MTG, Fookes M, Falush D, Keane JA, Parkhill J. Roary: rapid large-scale prokaryote pan genome analysis. Bioinformatics. 2015;31(22):3691.

19. Schmitz-Esser S, Gram L, Wagner M. Complete Genome Sequence of the Persistent Listeria monocytogenes Strain R479a. Genome Announc. 2015;3(2):e00150.

20. Gilmour MW, Graham M, Van Domselaar G, Tyler S, Kent H, Trout-Yakel KM, Larios O, Allen V, Lee B, Nadon C. High-throughput genome sequencing of two Listeria monocytogenes clinical isolates during a large foodborne outbreak. BMC Genomics. 2010;11(1):120.

21. Schmitz-Esser S, Müller A, Stessl B, Wagner M. Genomes of sequence type 121 Listeria monocytogenes strains harbor highly conserved plasmids and prophages. Front Microbiol. 2015;(6, 380):380.

22. DiMaio H. Listeria infection in women. Prim Care Update OB/GYNS. 2000; 7(1):40.

23. Charlier C, Goffinet F, Azria E, Leclercq A, Lecuit M. Inadequate management of pregnancy-associated listeriosis: lessons from four case reports. Clin Microbiol Infect. 2013;20(3):246.

24. Polin RA. Management of neonates with suspected or proven early-onset bacterial sepsis. Pediatrics. 2012;129(5):1006.

25. Fuchs A, Bielicki J, Mathur S, Sharland M, Van Den Anker J. Antibiotic Use for Sepsis in Neonates and Children: 2016 Evidence Update, vol. 2016: WHO Reviews. http://www.who.int/selection_medicines/committees/expert/21/ applications/s6_paed_antibiotics_appendix4_sepsis.pdf

26. Dong Y, Jiang SY, Zhou Q, Cao Y. Group B Streptococcus causes severe sepsis in term neonates: 8 years experience of a major Chinese neonatal unit. World J Pediatr. 2017;13(4):314

27. De Luca C, Donati L, D'Oria L, Licameli A, Pellegrino M, De Santis M. Listeria infection in pregnancy: a review of literature. Open Infect Dis J. 2015:9:2025
28. Temple ME, Nahata MC. Treatment of listeriosis. Ann Pharmacother. 2000; 34(5):656.

29. Lopez-Valladares G, Danielsson-Tham ML, Tham W. Implicated Food Products for Listeriosis and Changes in Serovars of Listeria monocytogenes Affecting Humans in Recent Decades. Foodborne Pathog Dis. 2018;15(7):387.

30. Smith AM, Tau NP, Smouse SL, Allam M, Ismail A, Ramalwa NR, Disenyeng B, Ngomane M, Thomas J. Outbreak of Listeria monocytogenes in South Africa, 2017-2018: Laboratory activities and experiences associated with whole-genome sequencing analysis of isolates. Foodborne Pathog Dis. 2019;16(7):524-530.

31. Elson R, Awofisayo-Okuyelu A, Greener T, Swift C, Painset A, Amar CFL, Newton A, Aird H, Swindlehurst M, Elviss N, et al. Utility of Whole Genome Sequencing To Describe the Persistence and Evolution of Listeria monocytogenes Strains within Crabmeat Processing Environments Linked to Two Outbreaks of Listeriosis. J Food Prot. 2019:82(1):30.

32. Duranti A, Sabbatucci M, Blasi G, Acciari VA, Ancora M, Bella A, Busani L, Centorame $P$, Camma C, Conti $F$, et al. A severe outbreak of listeriosis in central Italy with a rare pulsotype associated with processed pork products. J Med Microbiol. 2018;67(9):1351.

33. Schjorring S, Gillesberg Lassen S, Jensen T, Moura A, Kjeldgaard JS, Muller L, Thielke S, Leclerca A, Maury MM, Tourdjman M, et al. Cross-border outbreak of listeriosis caused by cold-smoked salmon, revealed by integrated surveillance and whole genome sequencing (WGS), Denmark and France, 2015 to 2017. Euro Surveill. 2017;22(50):1-5.

34. Ashrafi E, Alemzadeh A, Ebrahimi M, Ebrahimie E, Dadkhodaei N, Ebrahimi M. Amino acid features of P1B-ATPase heavy metal transporters enabling small numbers of organisms to cope with heavy metal pollution. Bioinform Biol Insights. 2011;5:S6206

35. Janto B, Ahmed A, Ito M, Liu J, Hicks DB, Pagni S, Fackelmayer OJ, Smith TA, Earl J, Elbourne LDH, et al. Genome of alkaliphilic Bacillus pseudofirmus OF4 reveals adaptations that support the ability to grow in an external $\mathrm{pH}$ range from 7.5 to 11.4. Environ Microbiol. 2011;13(12):3289.

36. Maury MM, Tsai Y-H, Charlier C, Touchon M, Chenal-Francisque V, Leclerca A, Criscuolo A, Gaultier C, Roussel S, Brisabois A, et al. Uncovering Listeria monocytogenes hypervirulence by harnessing its biodiversity. Nat Genet. 2016;48(3):308.

37. Fagerlund A, Langsrud S, Schirmer BCT, Møretrø T, Heir E. Genome Analysis of Listeria monocytogenes Sequence Type 8 Strains Persisting in Salmon and Poultry Processing Environments and Comparison with Related Strains. PLoS One. 2016;11(3):e0151117.

38. Naditz AL, Dzieciol M, Wagner M, Schmitz-Esser S. Plasmids contribute to food processing environment-associated stress survival in three Listeria monocytogenes ST121, ST8, and ST5 strains. Int J Food Microbiol. 2019;299: e0151117.

39. Wang H, Luo L, Zhang Z, Deng J, Wang Y, Miao Y, Zhang L, Chen X, Liu X, Sun $\mathrm{S}$, et al. Prevalence and molecular characteristics of Listeria monocytogenes in cooked products and its comparison with isolates from listeriosis cases. Front Med. 2018;12(1):104.

40. Luo L, Zhang Z, Wang H, Wang P, Lan R, Deng J, Miao Y, Wang Y, Wang Y, $\mathrm{Xu}$ J, et al. A 12-month longitudinal study of Listeria monocytogenes contamination and persistence in pork retail markets in China. Food Control. 2017;76:66.

41. Huang Y-T, Ko W-C, Chan Y-J, Lu J-J, Tsai H-Y, Liao C-H, Sheng W-H, Teng LJ, Hsueh P-R. Disease Burden of Invasive Listeriosis and Molecular Characterization of Clinical Isolates in Taiwan, 2000-2013. PLoS One. 2015; 10(11):e0141241.

\section{Publisher's Note}

Springer Nature remains neutral with regard to jurisdictional claims in published maps and institutional affiliations. 